\title{
Antiretroviral Drug Concentrations in Lymph Nodes: A Cross-Species Comparison of the Effect of Drug Transporter Expression, Viral Infection, and Sex in Humanized Mice, Nonhuman Primates, and Humans ${ }^{[}$
}

\author{
Erin Burgunder, DJohn K. Fallon, Nicole White, Amanda P. Schauer, Craig Sykes, \\ Leila Remling-Mulder, Martina Kovarova, Lourdes Adamson, Paul Luciw, J. Victor Garcia, \\ Ramesh Akkina, Philip C. Smith, and Angela D.M. Kashuba
}

Eshelman School of Pharmacy (E.B., J.K.F., N.W., A.P.S., C.S., P.C.S., A.D.M.K.) and School of Medicine (M.K., J.V.G., A.D.M.K.), University of North Carolina, Chapel Hill, North Carolina; School of Medicine, Colorado State University, Fort Collins, Colorado (L.R.-M., R.A.); and School of Medicine, University of California, Davis, California (L.A., P.L.)

Received April 24, 2019; accepted June 19, 2019

\section{ABSTRACT}

In a "kick and kill" strategy for human immunodeficiency virus (HIV) eradication, protective concentrations of antiretrovirals (ARVs) in the lymph node are important to prevent vulnerable cells from further HIV infection. However, the factors responsible for drug distribution and concentration into these tissues are largely unknown. Although humanized mice and nonhuman primates (NHPs) are crucial to HIV research, ARV tissue pharmacology has not been well characterized across species. This study investigated the influence of drug transporter expression, viral infection, and sex on ARV penetration within lymph nodes of animal models and humans. Six ARVs were dosed for 10 days in humanized mice and NHPs. Plasma and lymph nodes were collected at necropsy, 24 hours after the last dose. Human lymph node tissue and plasma from deceased patients were collected from tissue banks. ARV, active metabolite, and endogenous nucleotide concentrations were measured by liquid chromatography-tandem mass spectrometry, and drug transporter expression was measured using quantitative polymerase chain reaction and quantitative targeted absolute proteomics. In NHPs and humans, lymph node ARV concentrations were greater than or equal to plasma, and tenofovir diphosphate/ deoxyadenosine triphosphate concentration ratios achieved efficacy targets in lymph nodes from all three species. There was no effect of infection or sex on ARV concentrations. Low drug transporter expression existed in lymph nodes from all species, and no predictive relationships were found between transporter gene/protein expression and ARV penetration. Overall, common preclinical models of HIV infection were well suited to predict human ARV exposure in lymph nodes, and low transporter expression suggests primarily passive drug distribution in these tissues.

\section{SIGNIFICANCE STATEMENT}

During human immunodeficiency virus (HIV) eradication strategies, protective concentrations of antiretrovirals (ARVs) in the lymph node prevent vulnerable cells from further HIV infection. However, ARV tissue pharmacology has not been well characterized across preclinical species used for HIV eradication research, and the influence of drug transporters, HIV infection, and sex on ARV distribution and concentration into the lymph node is largely unknown. Here we show that two animal models of HIV infection (humanized mice and nonhuman primates) were well suited to predict human ARV exposure in lymph nodes. Additionally, we found that drug transporter expression was minimal and-along with viral infection and sex-did not affect ARV penetration into lymph nodes from any species.
This work was supported by the National Institutes of Health National Institute of Allergy and Infectious Diseases [Grants R01-AI111891 and P30AI50410 (to the University of North Carolina at Chapel Hill Center for AIDS Research)] and the National Institutes of Health Office of the Director [Grant P51-OD011107 (to the California National Primate Research Center)]. Its contents are solely the responsibility of the authors and do not necessarily represent the official view of the National Institutes of Health. Human tissue samples used in this study were provided by the National NeuroAIDS Tissue Consortium, which is funded by the National Institutes of Health National Institute of Neurological Disorders and Stroke and the National Institutes of Health National Institute of Mental Health [Grants U24-MH100931 (to the Manhattan HIV Brain Bank), U24-MH100930 (to Texas NeuroAIDS Research Center), U24-MH100929 (to the National Neurologic AIDS Bank), U24MH100928 (to the California NeuroAIDS Tissue Network), and U24MH100925 (to the National NeuroAIDS Tissue Consortium Data Coordinating Center)].

https://doi.org/10.1124/jpet.119.259150.

S This article has supplemental material available at jpet.aspetjournals.org.

\section{Introduction}

Advances in antiretroviral therapy (ART) potency and dosing have transformed human immunodeficiency virus (HIV) from a fatal diagnosis to a chronic condition, with patient life expectancy now comparable to that of an uninfected population (Samji et al., 2013). Despite this, challenges in treating and curing HIV remain (Lewin and Rouzioux, 2011). Although the majority of patients adherent to ART show undetectable viral replication in blood plasma, viral rebound occurs upon interruption of therapy (Chun et al., 1999). One theoretical cause of viral rebound is low-level viral replication within tissues (Natarajan et al., 1999; Buzón et al., 2011), caused by inadequate antiretroviral (ARV) penetration (Horiike et al., 2012; Fletcher et al., 2014, 2018). Low ARV 
penetration may also present challenges in protecting cells from infection during "kick and kill" strategies for HIV eradication (Lewin and Rouzioux, 2011).

In the area of HIV prevention, investigations into drug concentrations in colorectal and female genital tract tissues have shown highly variable ARV penetration (Dumond et al., 2007, 2009; Kwara et al., 2008; Else et al., 2011; Patterson et al., 2011; Thompson et al., 2015; Cottrell et al., 2016). However, considerably less drug exposure data are available for the putative lymph node reservoir, despite the fact that these tissues are central to the pathology of HIV (North et al., 2010; Dimopoulos et al., 2017). In the small number of published studies, results are conflicting. ARV lymph node concentrations range from 2-fold higher to 1000 -fold lower than those in plasma (Kinman et al., 2003; Solas et al., 2003; Fletcher et al., 2014). Furthermore, the methods used to investigate drug penetration in lymph nodes are also varied; some studies have used tissue homogenate (Solas et al., 2003), whereas others have isolated lymph node mononuclear cells (LNMCs) (Kinman et al., 2003; Fletcher et al., 2014).

Because human lymph node collection is invasive, preclinical models such as HIV-infected humanized mice and nonhuman primates (NHPs) with reverse transcriptasesimian/HIV may be informative for characterizing the pharmacokinetics of ARVs in lymph nodes. Preclinical models have been used to assess viral dynamics in tissues and curative ART strategies (Denton and García, 2011; Evans and Silvestri, 2013), but a cross-species comparison of ARV concentrations in putative tissue reservoirs is still needed. Furthermore, to understand concentration-response relationships of ARVs in tissues, it is important to investigate relevant biologic and disease-related factors that may influence these concentrations, such as drug transporters, viral infection, and sex (Antonelli et al., 1992; Speck et al., 2002; Fletcher et al., 2004; Giraud et al., 2010; Alam et al., 2016; Kis et al., 2016).

In this study, we aimed to quantify ARV concentrations and these aforementioned influencing factors using lymph nodes from humanized mice, NHPs, and deceased HIV-positive patients. We believe that these data improve our understanding of ARV pharmacology in the lymph node-an important but pharmacologically less characterized HIV tissue reservoir.

\section{Materials and Methods}

Animal Studies and Lymph Node Collection. A detailed description of the animal studies was published previously (Thompson et al., 2017) and is summarized here. This study used three common HIV preclinical models from two species: hu-HSC-Rag ( $\mathrm{RagHu}$ ) humanized mice $(n=36)$, bone marrow-liver-thymus (BLT) humanized mice $(n=13)$, and rhesus macaques (NHPs) $(n=18)$. All humanized mice and six NHPs were female. Aggressive interactions are common between male mice within the same cage, but isolating mice in separate cages can be inefficient and costly. For these reasons, we chose to use only female mice that could be safely kept in groups to minimize cage needs while maximizing animal well-being (Van Loo et al., 2003). RagHu mice $(n=18)$, BLT mice $(n=7)$, and NHPs $(n=10)$ were infected for 6 weeks with HIV $\mathrm{BAL}_{\mathrm{D} 7}, \mathrm{HIV}_{\mathrm{JRcsf}}$, and reverse transcriptase-simian/HIV, respectively (North et al., 2010). The remaining animals were uninfected (RagHu, $n=18$; BLT, $n=6$; and NHP, $n=8$ ). After the infection period, animals were dosed for 10 days to steady state and necropsy was performed 1 day after the last dose. Doses were chosen based on previously reported effective treatment regimens in these animal models (Denton et al., 2010; Neff et al., 2010; Shytaj et al., 2012; Massud et al., 2013; Veselinovic et al., 2014) and are reported in the Supplemental Methods. RagHu mice were dosed with efavirenz (EFV) only $(n=12)$, atazanavir (ATZ) only $(n=12)$, or a combination of tenofovir (TFV), emtricitabine (FTC), raltegravir (RAL), and maraviroc (MVC) $(n=12)$. All BLT mice received the five drug combination of TFV/FTC/RAL/MVC/ATZ $(n=13)$. Finally, NHPs were dosed with TFV/FTC/EFV/RAL $(n=9)$ or TFV/FTC/MVC/ATZ $(n=9)$. Further details on animal dosing, infection, and tissue collection can be found in the Supplemental Methods, Supplemental Fig. 1, and Supplemental Table 1.

At necropsy, plasma and lymph nodes were collected from all animals and snap frozen. For both RagHu and BLT mice, lymph node type was not determined; for NHPs, four lymph nodes (mesenteric, axillary, inguinal, and iliac) per animal were collected. Mouse and NHP tissue was divided for use in ARV and transporter expression analyses, with matched data obtained when possible (Supplemental Table 2). All animal studies were performed in accordance with Institutional Animal Care and Use Committee protocols from the University of North Carolina at Chapel Hill (protocol 15-168), Colorado State University (protocol 16-6998A), and the University of California, Davis (protocol 18345).

Human Lymph Node Collection. Human plasma and lymph node samples were obtained by the National Research Disease Interchange and the National NeuroAIDS Tissue Consortium from deceased HIV-positive patients who consented to organ donation prior to death or whose families consented to organ donation immediately after the patients' death. To screen for subjects who were adherent to ART or received ARV dosing premortem (and therefore most likely to have quantifiable tissue ARV concentrations), plasma samples were obtained based on desired subject characteristics such as tissue availability, viral load, and ART regimens containing TFV, FTC, EFV, RAL, MVC, or ATZ. Plasma samples were analyzed in our laboratory by liquid chromatography-tandem mass spectrometry (LC-MS/MS), and only subjects with measurable ARV plasma concentrations were included in the tissue request. Ultimately, lymph node sections from 13 subjects (two female) were requested, and each subject's ART regimen contained at least one drug of interest (eight TFV, four FTC, seven EFV, three RAL, two ATZ, and zero MVC). Human lymph node tissue was divided for use in ARV and transporter expression analyses, with matched data obtained when possible (Supplemental Table 2). Details on subject demographics, virology, ART dosing/regimens, and preliminary plasma ARV concentrations can be found in Supplemental Table 3.

ARV Concentration and Tissue Penetration Analyses. Concentrations of all six ARVs (TFV, FTC, EFV, RAL, MVC, and ATZ) were analyzed in plasma and lymph node tissue using LC-MS/MS. Additionally, the intracellular active metabolites tenofovir diphosphate (TFVdp) and emtricitabine triphosphate (FTCtp), and their respective endogenous nucleotides, deoxyadenosine triphosphate (dATP) and deoxycytidine triphosphate (dCTP), were analyzed in lymph node tissue.

\footnotetext{
ABBREVIATIONS: ART, antiretroviral therapy; ARV, antiretroviral; ATZ, atazanavir; BLQ, below the limit of quantification; BLT, bone marrow-liverthymus humanized mice; dATP, deoxyadenosine triphosphate; dCTP, deoxycytidine triphosphate; EC 90 , 90\% effective concentration; EFV, efavirenz; ENT1, equilibrative nucleoside transporter 1; FTC, emtricitabine; FTCtp, emtricitabine triphosphate; GAPDH, glyceraldehyde 3-phosphate dehydrogenase; HIV, human immunodeficiency virus; LC-MS/MS, liquid chromatography-tandem mass spectrometry; LLOD, lower limit of detection; LNMC, lymph node mononuclear cell; MRP, multidrug resistance-associated protein; MSI, mass spectrometry imaging; MVC, maraviroc; NHP, nonhuman primate; P-gp, P-glycoprotein; RagHu, hu-HSC-Rag; RAL, raltegravir; TFV, tenofovir; TFVdp, tenofovir diphosphate; TPR, tissue penetration ratio.
} 
Plasma and tissue samples were extracted by protein precipitation using stable, isotopically labeled internal standards. Extracts were analyzed by a Shimadzu high-performance liquid chromatography system with an API 5000 mass spectrometer (SCIEX, Framingham, MA) detector equipped with a TurboIonSpray interface. The lower limit of quantitation was $1 \mathrm{ng} / \mathrm{ml}$ for plasma and $0.002 \mathrm{ng} / \mathrm{ml}$ (FTC, MVC), $0.005 \mathrm{ng} / \mathrm{ml}$ (EFV, RAL, ATZ), $0.01 \mathrm{ng} / \mathrm{ml}$ (TFV), $1.11 \mathrm{ng} / \mathrm{ml}$ (dATP, dCTP), and $0.22 \mathrm{ng} / \mathrm{ml}$ (TFVdp, FTCtp) for tissue. Assay precision and accuracy was within $15 \%$.

At least $30 \mathrm{mg}$ lymph node tissue was homogenized using a Precellys Tissue Homogenizer (Bertin Technologies, Montigny-le-Bretonneux, France) in $1 \mathrm{ml}$ of 70:30 acetonitrile/ $1 \mathrm{mM}$ ammonium phosphate $(\mathrm{pH}$ 7.4) and extracted by protein precipitation as before. TFV and FTC were analyzed using a Waters Atlantis T3 $(50 \mathrm{~mm} \times 2.1 \mathrm{~mm}, 3 \mu \mathrm{m}$ particle size) column. EFV, RAL, MVC, and ATZ were separated using an Agilent Pursuit XRs 3 Diphenyl ( $50 \mathrm{~mm} \times 2 \mathrm{~mm}, 5 \mu \mathrm{m}$ particle size) high-performance liquid chromatography column. TFVdp, FTCtp, dATP, and dCTP were analyzed on a Thermo BioBasic AX column. Concentrations were converted from nanograms per milliliter to nanograms per gram based on a tissue density of $1.06 \mathrm{~g} / \mathrm{ml}$.

Tissue penetration ratios (TPRs) at the end of the dosing interval were calculated for each ARV by dividing lymph node concentrations by plasma concentrations. We also calculated active metabolite to endogenous nucleotide ratios, since the efficacy of the active metabolite depends on its concentration relative to the endogenous nucleotide it replaces during reverse transcription (Anderson et al., 2011).

Protein Expression Analysis. Protein concentrations of five efflux and three uptake transporters were measured by quantitative targeted absolute proteomics (Fallon et al., 2013). Transporters were chosen based on their relevance to ARV disposition, putative expression in lymph nodes, or previous investigation in other reservoir tissues (Supplemental Table 4) (Minuesa et al., 2011; Zhou et al., 2013; Nicol et al., 2014; Huang et al., 2016; Thompson et al., 2017). Approximately $100 \mathrm{mg}$ lymph node tissue was homogenized in $1.3 \mathrm{ml}$ hypotonic buffer [10 mM NaCl, $1.5 \mathrm{mM} \mathrm{MgCl} 2,10 \mathrm{mM}$ Tris $\mathrm{HCl}, \mathrm{pH}$ 7.4, and $150 \mu \mathrm{l}$ Complete Protease Inhibitor Solution (SigmaAldrich, St. Louis, MO)] using a Precellys Tissue Homogenizer, and 10-30 $\mu \mathrm{g}$ membrane protein was isolated as previously described (Fallon et al., 2016; Thompson et al., 2017). Membrane protein was dried down and reconstituted with $50 \mathrm{mM}$ ammonium bicarbonate buffer plus $40 \mathrm{mM}$ dithiothreitol, $10 \%$ sodium deoxycholate, and $10 \mu \mathrm{l}$ $\beta$-casein $(0.1 \mu \mathrm{g} / \mu \mathrm{l})$. Samples were then reduced for 40 minutes at $60^{\circ} \mathrm{C}$ followed by addition of $135 \mathrm{mM}$ iodoacetamide and incubation in the dark for 30 minutes at room temperature. One picomole of stable isotope labeled peptide standards (Theracode JPT Inc, Acton, MA) was added to the samples, followed by digestion with $25 \mu \mathrm{l}$ trypsin $(0.1 \mu \mathrm{g} / \mu \mathrm{l})$ at $37^{\circ} \mathrm{C}$ (Promega, Madison, WI). Digestion was interrupted after 18 hours with $10 \%$ trifluoroacetic acid, and samples were extracted using solid phase extraction on $33-\mu \mathrm{m}$ polymeric reversed phase extraction columns (Phenomenex, Torrance, CA). After final drydown and reconstitution in $98 \%$ formic acid $(0.1 \%)$ plus $2 \%$ acetonitrile, around $0.06-0.12 \mu \mathrm{g}$ microsomal protein was loaded onto a C18 trap column connected to a BEH130 C18 $(150 \mu \mathrm{m} \times 100 \mathrm{~mm}$, $1.7 \mu \mathrm{m}$ particle size) main separation column. Sample analysis was performed on a nanoACQUITY system (Waters, Milford, MA) coupled to a Qtrap 5500 mass spectrometer (SCIEX) equipped with a Nanospray III source. Analyst 1.5 and MultiQuant 2.0 software (SCIEX) were used for multiple reaction monitoring data acquisition and analysis. Peak area ratios of unlabeled/stable isotope labeled peptides were determined using the sum of two multiple reaction monitoring values. The lower limit of detection (LLOD) for the peptides was $0.1 \mathrm{pmol} / \mathrm{mg}$ protein, and $50 \mu \mathrm{g}$ NHP liver homogenate was used as a positive control.

Gene Expression Analysis. Gene expression of the same eight transporters was measured by quantitative polymerase chain reaction. Approximately $30 \mathrm{mg}$ tissue was homogenized using a Precellys Tissue Homogenizer and RNA was extracted using a Qiagen RNeasy Fibrous Tissue Mini Kit (Qiagen, Valencia, CA). The VILO Superscript cDNA Synthesis Kit (Thermo Fisher, Waltham, MA) was used to reverse transcribe $200 \mathrm{ng}$ RNA. A 10-cycle preamplification was performed, followed by 40 cycles of quantitative polymerase chain reaction using TaqMan primers and probes (Supplementary Table 5) on a QuantStudio 6 system (Life Technologies, Waltham, MA). Samples were run in triplicate, and transporter expression was normalized to the housekeeping gene glyceraldehyde 3-phosphate dehydrogenase (GAPDH) using the $2^{-\Delta \mathrm{CT}}$ method (Schmittgen and Livak, 2008). The LLOD was a 0.0001-fold change over GAPDH.

Statistical Analysis. In all species, ARV concentrations, gene expression values, or transporter protein concentrations below the limit of detection or below the limit of quantitation (BLQ) of the assay were imputed at half the lower limit of quantitation or LLOD, respectively. Relationships between plasma and lymph node drug concentrations were assessed using linear regression. Comparisons between species, infection status, and sex were performed using the Kruskal-Wallis one-way ANOVA with Dunn's method for multiple comparisons, and relationships between transporter expression and TPRs were investigated using multiple linear regression. Data were analyzed using SigmaPlot 13.0 (Systat Software Inc., San Jose, CA) with a significance level of $P<0.05$.

\section{Results}

There were no statistically significant differences in ARV concentrations between the four lymph nodes collected from each NHP (either uninfected or infected) (Supplemental Fig. 2 ), so data from all lymph nodes were averaged to generate one median value for each uninfected or infected animal. Plasma and lymph node ARV concentrations were not significantly different between BLT and RagHu mice, so data from both humanized mouse species were pooled for the remaining analyses (Supplemental Fig. 3). One female macaque dosed with TFV/FTC/EFV/RAL developed liver failure, resulting in drug concentrations 17- to 260-fold higher than other animals in the dosing group. This NHP was excluded from ARV concentration/penetration analyses but was included in drug transporter comparisons; transporter expression was not significantly different from other animals in the dosing group. Additionally, to avoid overestimating lymph node ARV penetration, mice and NHPs with BLQ plasma concentrations but measurable lymph node concentrations were excluded from analyses. These sample size adjustments are reflected in Supplemental Table 2.

ARV Concentrations in Plasma and Lymph Nodes. Several species-dependent trends in ARV concentrations and lymph node penetration relative to plasma were noted. Median ARV plasma concentrations were generally lowest in mice (14\% BLQ) and highest in humans, with a few exceptions: FTC and TFV were lowest in NHPs rather than mice, and RAL was highest in NHPs rather than humans; however, only one human plasma sample was available for RAL analysis (Fig. 1A). In lymph nodes, median ARV concentrations followed the same species trends seen in plasma, with $46 \%$ of mouse concentrations BLQ. The only notable differences were in TFV (highest in NHPs rather than humans) and RAL (highest in humans) (Fig. 1B). TPRs were calculated using matched lymph node and plasma concentrations. Median NHP lymph node TPRs ranged from 3-fold lower (RAL) to 25-fold higher (MVC) than plasma (Fig. 2). Compared with other species, NHP TPRs were highest for all drugs except FTC and RAL. Median TPRs ranged from 0.0002 (RAL) to 8 (FTC) for mice and from 0.8 (ATZ) to 2 (EFV) for humans. 
A

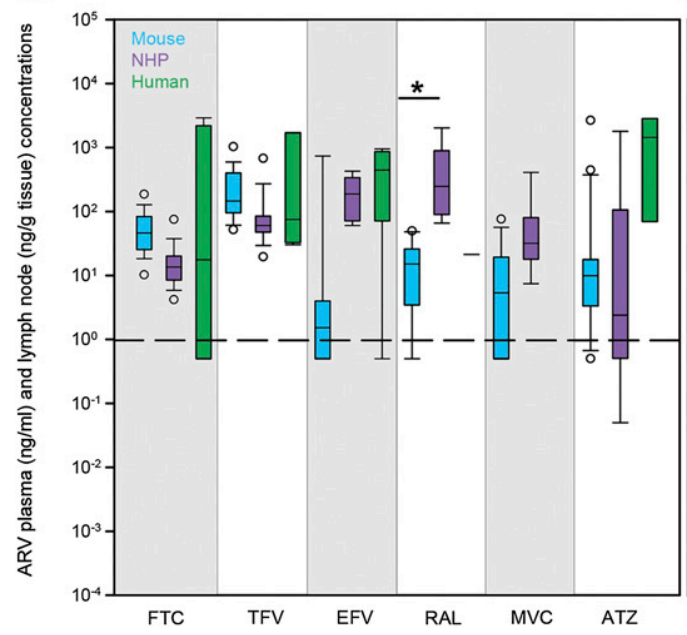

B

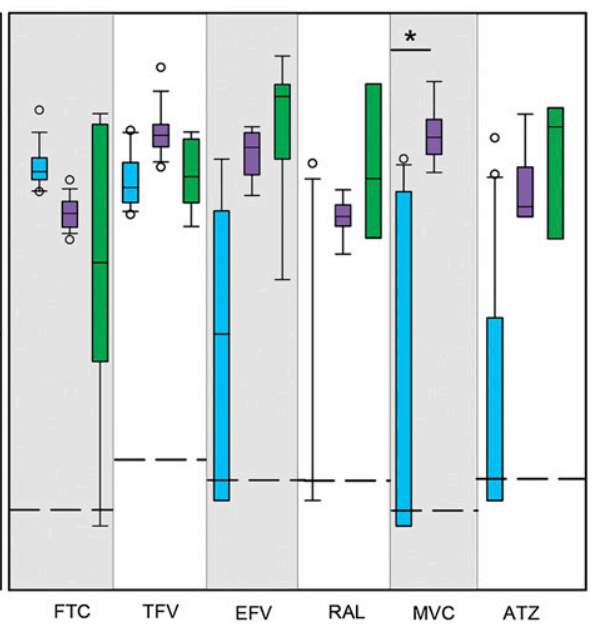

Fig. 1. Cross-species differences in ARV concentrations in plasma (A) (nanograms per milliliter) and lymph nodes (B) (nanograms per gram tissue) from mice (blue boxes), NHPs (purple boxes), and humans (green boxes). Boxes are the 25th percentile, median, and 75th percentile. Whiskers are the fifth and 95th percentiles, and dots are outliers. Dashed lines indicate the lower limit of quantitation. Sample sizes for each drug in each species are given in Supplemental Table 2. Betweenspecies differences were analyzed using Kruskal-Wallis one-way ANOVA with Dunn's method correction for multiple comparisons. $* P<0.05$.
Excluding BLQ concentrations in mice, there were several significant relationships between lymph node and plasma concentrations: FTC $\left(r^{2}=0.73, P<0.001\right)$, TFV $\left(r^{2}=0.03\right)$, $\operatorname{EFV}\left(r^{2}=0.73, P<0.001\right), \operatorname{RAL}\left(r^{2}=0.004\right), \operatorname{MVC}\left(r^{2}=0.40\right.$, $P<0.05)$, and ATZ $\left(r^{2}=0.55, P<0.01\right)$.

Active Metabolite and Endogenous Nucleotide Concentrations and Ratios. Active metabolite concentrations showed greater between-species variability than parent drugs, particularly for TFVdp. Similar to the pattern of TFV TPRs, median TFVdp concentrations were highest in NHPs (Fig. 3A). On a molar basis, lymph nodes from all species contained more parent drugs than active metabolites, but NHPs had the greatest proportion of FTC converted to FTCtp $(4.8 \%$; mouse $=0.2 \%$, human $=1.1 \%)$ and $\mathrm{TFV}$ converted to

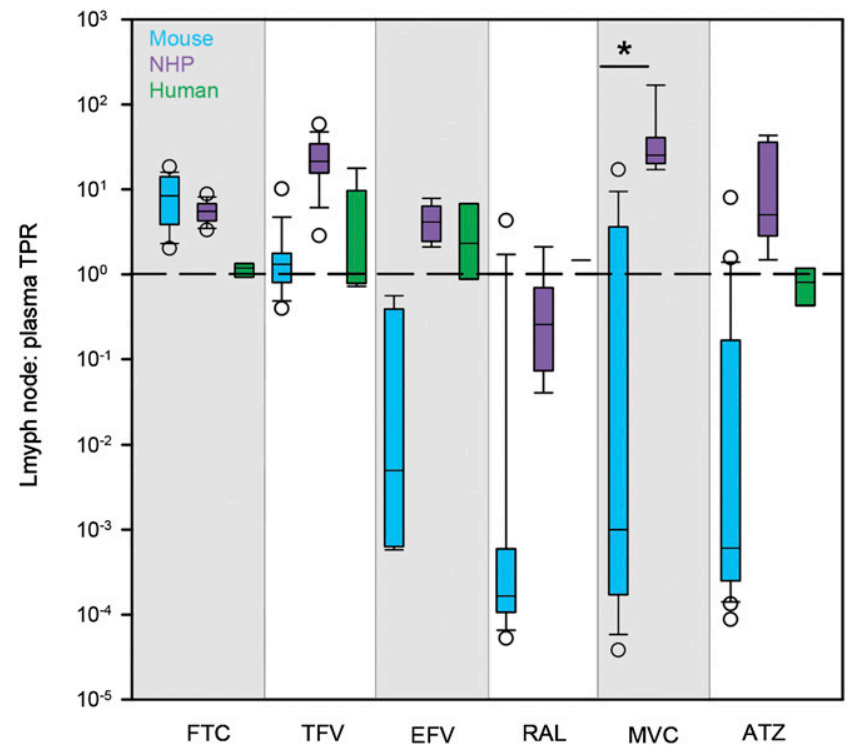

Fig. 2. Cross-species differences in ARV TPRs in mice (blue boxes), NHPs (purple boxes), and humans (green boxes). Boxes are the 25th percentile, median, and 75th percentile. Whiskers are the fifth and 95th percentiles, and dots are outliers. The dashed line indicates equal drug concentrations in lymph node and plasma. Sample sizes for each drug in each species are given in Supplemental Table 2. Between-species differences were analyzed using Kruskal-Wallis one-way ANOVA with Dunn's method correction for multiple comparisons. $* P<0.05$.
TFVdp $(6.4 \%$; mouse $=0.1 \%$, human $=0.2 \%)($ Table 1$)$. Concentrations of the endogenous nucleotides dCTP and dATP were highest in NHP lymph nodes but were not significantly different across species (Fig. 3A). When comparing endogenous nucleotides to corresponding active metabolites, only NHP dATP concentrations were significantly different from TFVdp concentrations $(P<0.05)$. Therefore, after normalizing active metabolite concentrations to endogenous nucleotides, TFVdp/dATP ratios were significantly higher in NHPs, but FTCtp/dCTP ratios were similar across species (Fig. 3B).

Effect of Viral Infection and Sex on ARV Penetration in Lymph Nodes. No differences in lymph node drug penetration due to viral infection (Fig. 4) or sex (Fig. 5) were found in any species. There were also no trends in the variability of ARV penetration between uninfected and infected animals or males and females.

Drug Transporter Expression in Lymph Nodes. Lymph node drug transporter expression was low across all species. Gene expression of all eight transporters was consistently lower than the housekeeping gene GAPDH in both mice and NHPs (Fig. 6A). Median equilibrative nucleoside transporter 1 (ENT1) gene expression was highest overall for both species. Mouse gene expression was higher than NHP for all transporters but multidrug resistance-associated protein 2 (MRP2). Transporter protein expression was also low in NHP and human lymph nodes; breast cancer resistance protein, ENT1, and organic cation transporter 3 were the only NHP transporters with median concentrations above the limit of detection $(94 \%, 100 \%$, and $94 \%$ of samples above LLOD, respectively), and median concentrations of all eight transporters were below the limit of detection in humans (Fig. 6B)

Effect of Drug Transporter Expression on ARV Lymph Node Penetration. Few predictive relationships were found between ARV TPRs and drug transporter expression. Higher mouse and NHP gene expression of MRP2 was predictive of greater TFV penetration in lymph nodes $\left(P=0.002, r^{2}=0.27\right)$ (Supplementary Table 6$)$, and greater ATZ penetration in NHPs and humans was predicted by an increase in combined MRP1 and P-glycoprotein (P-gp) protein expression $\left(P<0.001, r^{2}=0.99\right)$ (Supplementary Table 7). 
A
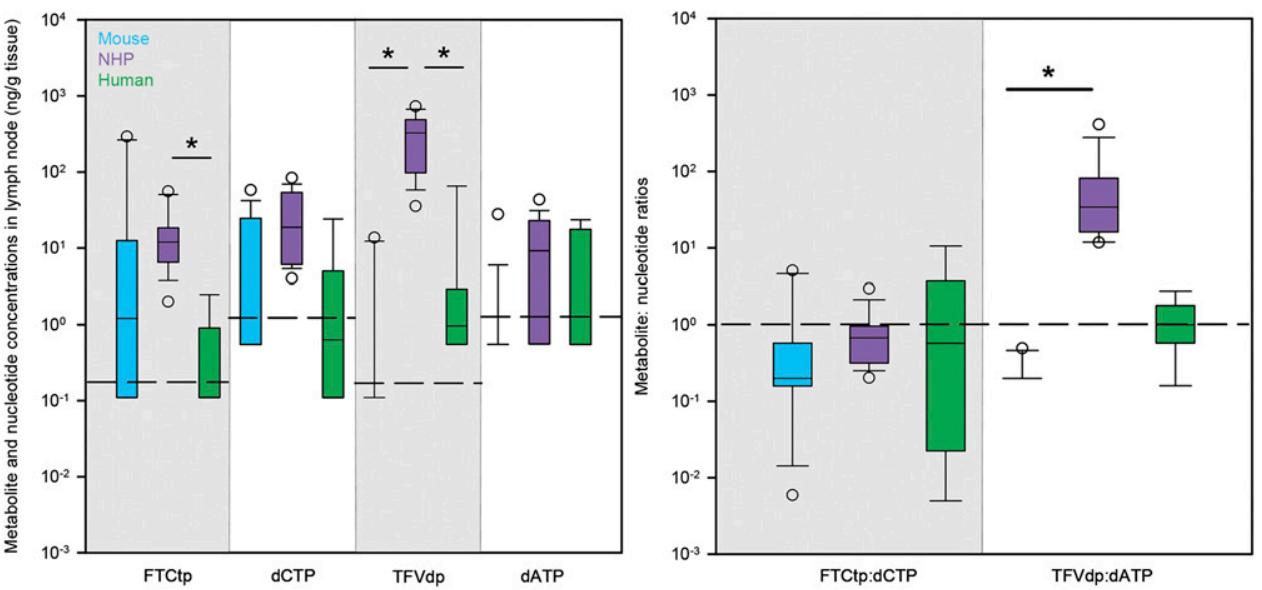

Fig. 3. Cross-species differences in active metabolite and endogenous nucleotide concentrations (nanograms per gram tissue) (A) and metabolite/nucleotide ratios (B) in lymph nodes of mice (blue boxes), NHPs (purple boxes), and humans (green boxes). Boxes are the 25th percentile, median, and 75th percentile. Whiskers are the fifth and 95th percentiles, and dots are outliers. The dashed line in (A) indicates lower limits of quantitation, whereas the dashed line in (B) indicates equal metabolite and nucleotide concentrations. Sample sizes for each drug in each species are given in Supplemental Table 2. Between-species differences were analyzed using Kruskal-Wallis one-way ANOVA with Dunn's method correction for multiple comparisons. ${ }^{*} P<0.05$.

\section{Discussion}

This study is the first to evaluate ARV exposure in lymph nodes of three species. In this investigation, we determined that there was no effect of acute HIV infection or sex on ARV lymph node concentrations. However, we did find several interesting species-dependent differences in drug exposure. In mice, EFV, RAL, MVC, and ATZ penetration was low and variable: between $33 \%$ (EFV) and $83 \%$ (RAL) of lymph node concentrations were BLQ. No relationship was found between the amount of mouse lymph node analyzed and tissue concentrations, indicating that BLQ concentrations are not the result of small tissue quantities and LC-MS/MS sensitivity. There were also no animal-specific trends in tissue concentrations: previous analyses found no BLQ concentrations in brain tissue from any mouse, whereas $96 \%$ of ileum and $87 \%$ of rectum concentrations were BLQ in the same animals (Thompson, 2017; Srinivas et al., 2018). When considering only mice with quantifiable lymph node concentrations, drug penetration was generally similar between mice, NHPs, and humans.

Similar to the pattern of TFV penetration ratios, TFVdp concentrations and TFVdp/dATP ratios were significantly higher in NHPs. Previous work in NHPs and humans has shown that in target lymphoid organs, the concentration ratio of TFVdp to the endogenous substrate dATP may be a better predictor of efficacy than TFVdp concentrations alone (GarcíaLerma et al., 2011; Cottrell et al., 2016). Using an in vitro enzymatic inhibition assay, investigators have shown that a TFVdp/dATP ratio $\geq 1$ corresponds to $100 \%$ reverse transcriptase inhibition in NHPs (García-Lerma et al., 2011). In our study, median NHP TFVdp/dATP ratios were 34, well above an efficacy target of 1 . Similarly, a predictive pharmacokinetic/pharmacodynamic model in humans found

TABLE 1

Molar percentage of FTC, FTCtp, TFV, and TFVdp in mouse, NHP, and human lymph nodes Data are presented as the median (minimum, maximum) molar percentage.

\begin{tabular}{lccc}
\hline ARV & Mouse & NHP & Human \\
\hline FTC & $99.8(77.4,100)$ & $95.2(79,100)$ & $98.9(2.1,100)$ \\
FTCtp & $0.2(0,22.6)$ & $4.8(0,21)$ & $1.1(0,97.9)$ \\
TFV & $99.9(95.6,100)$ & $93.6(64.2,100)$ & $99.8(89.3,100)$ \\
TFVdp & $0.1(0,4.4)$ & $6.4(0,35.8)$ & $0.2(0,10.7)$ \\
\hline
\end{tabular}

a $90 \%$ effective TFVdp/dATP concentration ratio $\left(\mathrm{EC}_{90}\right)$ of 0.29 for protecting $\mathrm{CD} 4+\mathrm{T}$ cells from viral challenge (Cottrell et al., 2016); in our study, this efficacy target was exceeded by NHPs and humans and almost achieved in mice (median ratios of 34,1 , and 0.2 , respectively). These data show that despite a species difference in TFVdp concentrations, both preclinical species meet previously investigated human TFVdp/dATP efficacy targets in lymph node tissue.

A previous study reported that TFVdp concentrations in human LNMCs were significantly lower than in peripheral blood mononuclear cells and that these low concentrations were associated with HIV replication (Fletcher et al., 2014). Indeed, it is well known that even during suppressive ART, viral RNA and DNA can persist in lymph nodes and other tissues from NHPs and humans (North et al., 2010; Deleage et al., 2016; Lamers et al., 2016; Estes et al., 2017), and there may be evidence of ongoing HIV replication within the lymph nodes of ART-suppressed patients (Lorenzo-Redondo et al., 2016; Halvas et al., 2019). However, in contrast to previous LNMC studies-which do not account for drug lost during cell isolation and may underestimate concentrations-we found that lymph node TFV concentrations are higher than or equal to plasma, and TFVdp/dATP ratios meet or exceed the $\mathrm{EC}_{90}$ target to inhibit HIV reverse transcription.

Yet the concentrations we have presented are an averaged concentration throughout the lymph node. It has been previously demonstrated that drug distribution within a tissue can be variable (Fischman et al., 1998), and we have shown heterogeneous EFV concentrations across five different putative tissue reservoirs using mass spectrometry imaging (MSI) (Thompson et al., 2015). Recently, we also saw little colocalization of viral RNA with efficacious ARV concentrations in the brain (3\% overlap) (Srinivas et al., 2018). Therefore, an ARV's location within tissue is as important as overall concentration. We are continuing to evaluate lymph node distribution by quantitative MSI to address this issue.

In addition to ARV concentrations, this study was also the first to examine cross-species expression of relevant ARV transporters in lymph nodes. Overall, transporter expression in lymph nodes is comparable to what we have seen in brain tissue (Srinivas, 2018) but lower than other HIV reservoirs such as the testis, ileum, and rectum (Huang et al., 2016; Thompson et al., 2017). The most highly expressed 
A

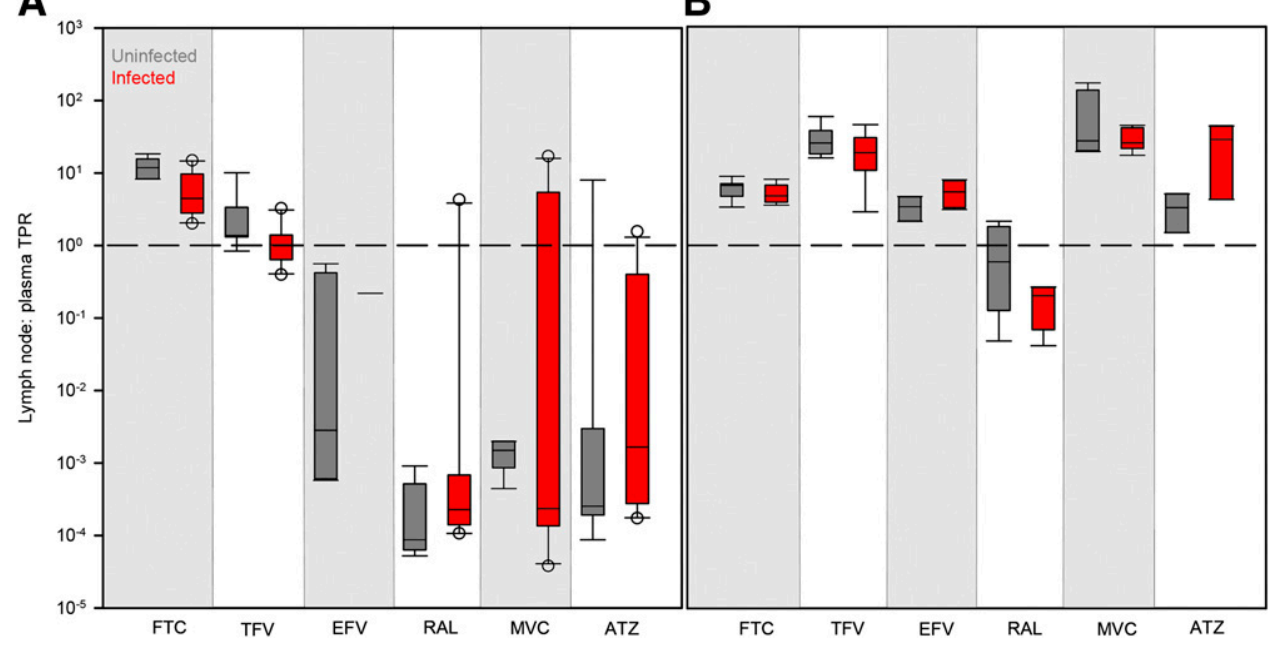

Fig. 4. Differences in ARV TPRs in uninfected (gray boxes) and infected (red boxes) mouse (A) and NHP (B) lymph nodes. Boxes are the 25th percentile, median, and 75th percentile. Whiskers are the fifth and 95th percentiles, and dots are outliers. The dashed line indicates equal drug concentrations in lymph node and plasma. Sample sizes for each drug in each species are given in Supplemental Table 2. Infection differences were analyzed using Kruskal-Wallis one-way ANOVA with Dunn's method correction for multiple comparisons. transporter by gene and protein methods was ENT1. This aligns with previous RNA expression and immunocytochemistry data for ENT1 in lymphoblastic T-cell lines and CD4+ $\mathrm{T}$ cells isolated from HIV-negative donors (Minuesa et al., 2008). ENT1 is responsible for the cellular uptake of nucleosides such as adenosine and uridine, which are important for immunostimulatory responses and lymphocyte proliferation (Goodman and Weigle, 1983, 1984); therefore, increased ENT1 expression in lymph nodes is not unexpected.

To determine whether even low-level transporter expression could influence drug distribution in the lymph node, we used multilinear regression to assess relationships between ARV penetration ratios and transporter gene/protein expression across species, including any potential link between ENT1 expression and TFV (an adenosine analog) (Viread, 2001). Although the positive associations between TFV versus MRP2 (in mice and NHPs) and ATZ versus MRP1+P-gp (in NHPs and humans) are significant, these relationships were primarily driven by the number of below the limit of detection transporter concentrations and are inconsistent with the intracellular-to-extracellular direction of MRP1, MRP2, and P-gp efflux transporters. Taken together, generally low drug transporter expression in the lymph node, and lack of physiologically relevant predictive relationships between transporters and ARV penetration, suggests that drug distribution in the lymph node may occur by passive, diffusion-based mechanisms-contributing to the heterogeneous ARV distribution we have seen in the lymph node for EFV (Thompson et al., 2015).

There are several limitations in our assessment of ARV tissue penetration. One important consideration is our use of steady-state plasma and tissue concentrations obtained at the end of the dosing interval to estimate TPRs. This approach may overestimate drug penetration into lymph nodes, since the difference in drug concentrations in these two compartments is likely greatest at the end of the dosing interval. A better method of estimating tissue penetration is by dividing lymph node and plasma areas under the concentration time curves $\left(\mathrm{AUC}_{\text {tissue }} / \mathrm{AUC}_{\text {plasma }}\right)$, which can be determined by repeated tissue sampling. However, in our animal studies, we opted to start collecting a set of whole lymph nodes at the end of the dosing interval. A second possible limitation is blood contamination within lymph node homogenate, although no blood was observed during visual inspection of the tissue.
A

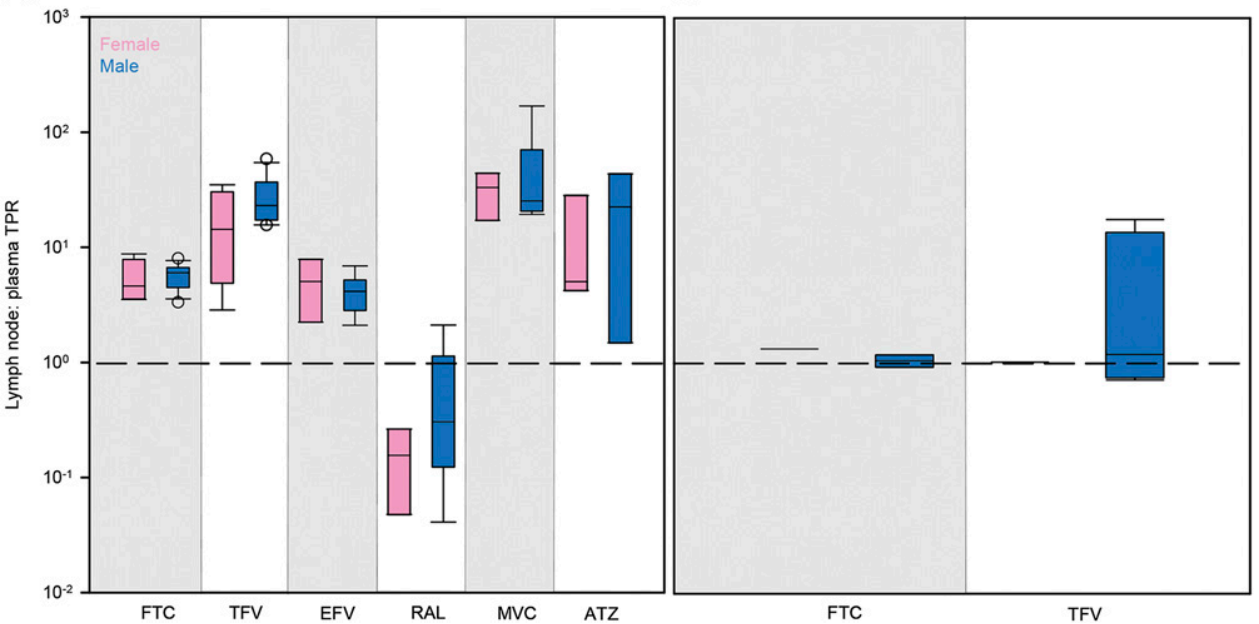

Fig. 5. Differences in ARV TPRs in female (pink boxes) and male (blue boxes) NHP (A) and human (B) lymph nodes. Boxes are the 25th percentile, median, and 75 th percentile. Whiskers are the fifth and 95th percentiles, and dots are outliers. Dashed lines indicate equal drug concentrations in lymph node and plasma. Sample sizes for each drug in each species are given in Supplemental Table 2. Sex differences were analyzed using KruskalWallis one-way ANOVA with Dunn's method correction for multiple comparisons. 
A

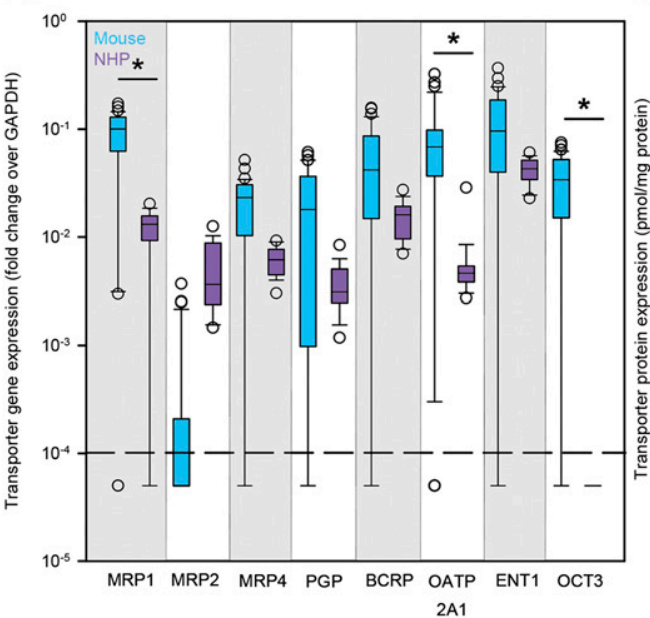

B

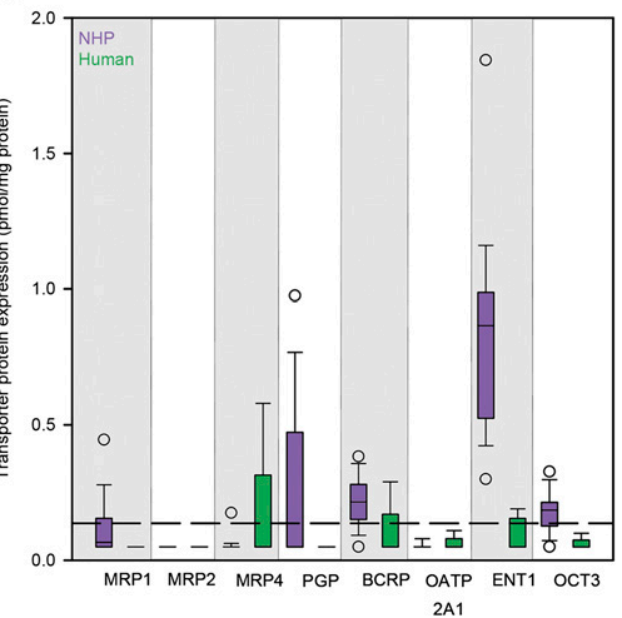

Fig. 6. Cross-species differences in drug transporter expression in lymph nodes. (A) Gene expression (measured as the fold change over the housekeeping gene GAPDH) of eight drug transporters in the lymph nodes of mice (blue boxes) and NHPs (purple boxes). (B) Protein concentrations (picomoles per milligram protein) of eight drug transporters in the lymph nodes of NHPs (purple boxes) and humans (green boxes). Boxes are the 25 th percentile, median, and 75th percentile. Whiskers are the fifth and 95th percentiles, and dots are outliers. Dashed lines in (A) and (B) represent the lower limit of quantitation and the LLOD, respectively. Sample sizes for each species are given in Supplemental Table 2. Between-species differences were analyzed using Kruskal-Wallis one-way ANOVA with Dunn's method correction for multiple comparisons. ${ }^{*} P<0.05$
Using MSI we have analyzed lymph node sections for heme (a blood marker) and ARVs and found little colocalization of the two ( $\leq 25 \%$ overlap) (unpublished data). We will continue these analyses in the future to correct for blood contamination in the lymph node. Finally, lymph node ARV concentrations reported here include both protein bound and unbound fractions. Preliminary studies in a small set of NHP samples suggest that RAL, MVC, and ATZ binding is minimal in lymph nodes ( $<50 \%$ bound), whereas EFV binding is comparable to plasma ( $\sim 97 \%$ bound) (unpublished data). Further binding analyses are ongoing.

Additionally, there were several limitations to our drug transporter analyses. We were unable to compare all tissues for gene and protein expression. Although protein expression is a more relevant measure of transporter presence in tissue (Ohtsuki et al., 2012), the extremely small size (15-30 mg) of the mouse lymph nodes was prohibitive for transporter protein analyses, which requires at least $100 \mathrm{mg}$ tissue before initial homogenization. Therefore, we opted to evaluate gene expression in mice and NHPs. Since transporter expression was low across all species by both methods, it is unlikely that any appreciable transporter protein concentrations would have been found in mice even with an adequate supply of tissue. Also, our gene and protein analyses do not account for transporter activity. Future immunohistochemistry analysis of transporter localization in lymph nodes will investigate whether the majority of transporters are localized to plasma membranes, nuclear membranes, or within cytosol (where they would be functionally inactive) (Giacomini et al., 2010).

In conclusion, we measured ARV penetration and drug transporter expression in humanized mouse, NHP, and human lymph nodes to further characterize species differences in ARV pharmacology within this putative HIV reservoir. We also investigated the effect of infection status and sex on ARV penetration, and we characterized the relationship between drug transporter expression and ARV penetration in the lymph node. Most notably, in NHPs and humans we found that lymph node ARV exposure was greater than or equal to plasma, and lymph node concentrations (with the exception of TFV and RAL) were well predicted by those in plasma. We also found that mouse and NHP TFVdp/dATP ratios met or exceeded human $\mathrm{EC}_{90}$ efficacy targets in lymph nodes. There was no effect of infection or sex on lymph node ARV penetration in any species. We also found low drug transporter expression in lymph nodes from all species and no predictive relationships between transporter gene or protein expression and ARV exposure, indicating that drug distribution in lymph nodes may occur through passive mechanisms. Our future research will focus on other physiologic (protein binding, tissue fibrosis) or physiochemical (molecular weight, lipophilicity) factors that may contribute to altered drug penetration and distribution in this reservoir. Along with the findings presented here, these data will have important implications for future preclinical and clinical drug development studies of HIV therapies targeted to lymph nodes.

\section{Acknowledgments}

We acknowledge the National Research Disease Interchange and we thank Dr. Howard Fox and Elizabeth Kulka of the National NeuroAIDS Tissue Consortium for providing the human tissue samples used in this study.

\section{Authorship Contributions}

Participated in research design: Fallon, Luciw, Garcia, Akkina, Smith, Kashuba.

Conducted experiments: Burgunder, Fallon, White, Schauer, Sykes, Remling-Mulder, Kovarova, Adamson, Luciw, Akkina.

Contributed new reagents or analytic tools: Smith.

Performed data analysis: Burgunder, Garcia.

Wrote or contributed to the writing of the manuscript: Burgunder, Kashuba.

\section{References}

Alam C, Whyte-Allman S-K, Omeragic A, and Bendayan R (2016) Role and modulation of drug transporters in HIV-1 therapy. Adv Drug Deliv Rev 103:121-143.

Anderson PL, Kiser JJ, Gardner EM, Rower JE, Meditz A, and Grant RM (2011)

Pharmacological considerations for tenofovir and emtricitabine to prevent HIV infection. J Antimicrob Chemother 66:240-250.

Antonelli G, Turriziani O, Cianfriglia M, Riva E, Dong G, Fattorossi A, and Dianzani F (1992) Resistance of HIV-1 to AZT might also involve the cellular expression of multidrug resistance P-glycoprotein. AIDS Res Hum Retroviruses 8:1839-1844.

Buzón MJ, Codoñer FM, Frost SDW, Pou C, Puertas MC, Massanella M, Dalmau J, Llibre JM, Stevenson M, Blanco J, et al. (2011) Deep molecular characterization of HIV-1 dynamics under suppressive HAART. PLoS Pathog 7:e1002314.

Chun TW, Davey RT Jr, Engel D, Lane HC, and Fauci AS (1999) Re-emergence of HIV after stopping therapy. Nature 401:874-875.

Cottrell ML, Yang KH, Prince HMA, Sykes C, White N, Malone S, Dellon ES, Madanick RD, Shaheen NJ, Hudgens MG, et al. (2016) A translational pharmacology approach to predicting outcomes of preexposure prophylaxis against HIV in men and women using tenofovir disoproxil fumarate with or without emtricitabine. $J$ Infect Dis 214:55-64. 
Deleage C, Wietgrefe SW, Del Prete G, Morcock DR, Hao XP, Piatak M Jr, Bess J, Anderson JL, Perkey KE, Reilly C, et al. (2016) Defining HIV and SIV reservoirs in lymphoid tissues. Pathog Immun 1:68-106.

Denton PW and García JV (2011) Humanized mouse models of HIV infection. AIDS Rev 13:135-148.

Denton PW, Krisko JF, Powell DA, Mathias M, Kwak YT, Martinez-Torres F, Zou W Payne DA, Estes JD, and Garcia JV (2010) Systemic administration of antiretrovirals prior to exposure prevents rectal and intravenous HIV-1 transmission in humanized BLT mice. PLoS One 5:e8829.

Dimopoulos Y, Moysi E, and Petrovas C (2017) The lymph node in HIV pathogenesis. Curr HIV/AIDS Rep 14:133-140.

Dumond JB, Patterson KB, Pecha AL, Werner RE, Andrews E, Damle B, Tressler R Worsley J, and Kashuba ADM (2009) Maraviroc concentrates in the cervicovaginal fluid and vaginal tissue of HIV-negative women. J Acquir Immune Defic Syndr $\mathbf{5 1}$ 546-553.

Dumond JB, Yeh RF, Patterson KB, Corbett AH, Jung BH, Rezk NL, Bridges AS, Stewart PW, Cohen MS, and Kashuba ADM (2007) Antiretroviral drug exposure in the female genital tract: implications for oral pre- and post-exposure prophylaxis. AIDS 21:1899-1907.

Else LJ, Taylor S, Back DJ, and Khoo SH (2011) Pharmacokinetics of antiretroviral drugs in anatomical sanctuary sites: the male and female genital tract. Antivir Ther 16:1149-1167.

Estes JD, Kityo C, Ssali F, Swainson L, Makamdop KN, Del Prete GQ, Deeks SG, Luciw PA, Chipman JG, Beilman GJ, et al. (2017) Defining total-body AIDS-virus burden with implications for curative strategies. Nat Med 23: 1271-1276.

Evans DT and Silvestri G (2013) Nonhuman primate models in AIDS research. Curr Opin HIV AIDS 8:255-261.

Fallon JK, Neubert H, Hyland R, Goosen TC, and Smith PC (2013) Targeted quantitative proteomics for the analysis of 14 UGT1As and -2Bs in human liver using NanoUPLC-MS/MS with selected reaction monitoring. J Proteome Res 12 $4402-4413$

Fallon JK, Smith PC, Xia CQ, and Kim M-S (2016) Quantification of four efflux drug transporters in liver and kidney across species using targeted quantitative proteomics by isotope dilution NanoLC-MS/MS. Pharm Res 33 2280-2288.

Fischman AJ, Babich JW, Bonab AA, Alpert NM, Vincent J, Callahan RJ, Correia JA and Rubin RH (1998) Pharmacokinetics of [18F]trovafloxacin in healthy human subjects studied with positron emission tomography. Antimicrob Agents Chemother 42:2048-2054.

Fletcher CV, Jiang H, Brundage RC, Acosta EP, Haubrich R, Katzenstein D, and Gulick RM (2004) Sex-based differences in saquinavir pharmacology and virologic response in AIDS Clinical Trials Group Study 359. J Infect Dis 189: 1176-1184.

Fletcher CV, Staskus K, Wietgrefe SW, Rothenberger M, Reilly C, Chipman JG, Beilman GJ, Khoruts A, Thorkelson A, Schmidt TE, et al. (2014) Persistent HIV-1 replication is associated with lower antiretroviral drug concentrations in lymphatic tissues. Proc Natl Acad Sci USA 111:2307-2312.

Fletcher CV, Thorkelson A, Winchester L, Mykris T, Weinhold J, Campbell K, Anderson J, Zulk J, Moshele P, and Schacker T (2018) Comparative lymphoid tissue pharmacokinetics (PK) of integrase inhibitors, INSTI. Conference on Retroviruses and Opportunistic Infections; 2018 Mar 4-7; Boston.

García-Lerma JG, Aung W, Cong ME, Zheng Q, Youngpairoj AS, Mitchell J, Holder A, Martin A, Kuklenyik S, Luo W, et al. (2011) Natural substrate concentrations can modulate the prophylactic efficacy of nucleotide HIV reverse transcriptase inhibitors. $J$ Virol 85:6610-6617.

Giacomini KM, Huang SM, Tweedie DJ, Benet LZ, Brouwer KL, Chu X, Dahlin A Evers R, Fischer V, Hillgren KM, et al.; International Transporter Consortium (2010) Membrane transporters in drug development. Nat Rev Drug Discov 9 215-236.

Giraud C, Manceau S, and Treluyer J-M (2010) ABC transporters in human lymphocytes: expression, activity and role, modulating factors and consequences for antiretroviral therapies. Expert Opin Drug Metab Toxicol 6 $571-589$

Goodman MG and Weigle WO (1983) Activation of lymphocytes by a thiol-derivatized nucleoside: characterization of cellular parameters and responsive subpopulations. $J$ Immunol 130:551-557.

Goodman MG and Weigle WO (1984) Intracellular lymphocyte activation and carriermediated transport of C8-substituted guanine ribonucleosides. Proc Natl Acad Sci USA 81:862-866.

Halvas E, Joseph K, Brandt LD, Botha JC, Sobolewski M, Jacobs JL, Keele BF Kearney MF, Coffin JM, Rausch JW, et al. (2019) Nonsuppressible ciremia on ART from large cell clones carrying intact proviruses. Conference on Retroviruses and Opportunistic Infections; 2019 Mar 4-7; Boston.

Horiike M, Iwami S, Kodama M, Sato A, Watanabe Y, Yasui M, Ishida Y, Kobayashi T, Miura T, and Igarashi T (2012) Lymph nodes harbor viral reservoirs that cause rebound of plasma viremia in SIV-infected macaques upon cessation of combined antiretroviral therapy. Virology 423:107-118.

Huang Y, Hoque MT, Jenabian M-A, Vyboh K, Whyte S-K, Sheehan NL, Brassard P, Bélanger M, Chomont N, Fletcher CV, et al. (2016) Antiretroviral drug transporters and metabolic enzymes in human testicular tissue: potential contribution to HIV-1 sanctuary site. J Antimicrob Chemother 71 1954-1965.

Kinman L, Brodie SJ, Tsai CC, Bui T, Larsen K, Schmidt A, Anderson D, Morton WR Hu S-L, and Ho RJY (2003) Lipid-drug association enhanced HIV-1 protease inhibitor indinavir localization in lymphoid tissues and viral load reduction: a proof of concept study in HIV-2287-infected macaques. J Acquir Immune Defic Syndr 34: 387-397.

Kis O, Sankaran-Walters S, Hoque MT, Walmsley SL, Dandekar S, and Bendayan R (2016) HIV-1 alters intestinal expression of drug transporters and metabolic enzymes: implications for antiretroviral drug disposition. Antimicrob Agents Chemother 60:2771-2781.

Kwara A, Delong A, Rezk N, Hogan J, Burtwell H, Chapman S, Moreira CC, Kurpewski J, Ingersoll J, Caliendo AM, et al. (2008) Antiretroviral drug concentrations and HIV RNA in the genital tract of HIV-infected women receiving long-term highly active antiretroviral therapy. Clin Infect Dis $\mathbf{4 6}$ $719-725$

Lamers SL, Rose R, Maidji E, Agsalda-Garcia M, Nolan DJ, Fogel GB, Salemi M, Garcia DL, Bracci P, Yong W, et al. (2016) HIV DNA is frequently present within pathologic tissues evaluated at autopsy from combined antiretrovira therapy-treated patients with undetectable viral loads. $J$ Virol 90: 8968-8983.

Lewin SR and Rouzioux C (2011) HIV cure and eradication: how will we get from the laboratory to effective clinical trials? AIDS 25:885-897.

Lorenzo-Redondo R, Fryer HR, Bedford T, Kim E-Y, Archer J, Pond SLK, Chung Y-S, Penugonda S, Chipman J, Fletcher CV, et al. (2016) Persistent HIV-1 replication maintains the tissue reservoir during therapy. Nature 530:51-56.

Massud I, Aung W, Martin A, Bachman S, Mitchell J, Aubert R, Solomon Tsegaye T, Kersh E, Pau C-P, Heneine W, et al. (2013) Lack of prophylactic efficacy of ora maraviroc in macaques despite high drug concentrations in rectal tissues. $J$ Virol 87:8952-8961.

Minuesa G, Huber-Ruano I, Pastor-Anglada M, Koepsell H, Clotet B, and MartinezPicado J (2011) Drug uptake transporters in antiretroviral therapy. Pharmacol Ther 132:268-279.

Minuesa G, Purcet S, Erkizia I, Molina-Arcas M, Bofill M, Izquierdo-Useros N, Casado FJ, Clotet B, Pastor-Anglada M, and Martinez-Picado J (2008) Expression and functionality of anti-human immunodeficiency virus and anticancer drug uptake transporters in immune cells. J Pharmacol Exp Ther 324: $558-567$.

Natarajan V, Bosche M, Metcalf JA, Ward DJ, Lane HC, and Kovacs JA (1999) HIV-1 replication in patients with undetectable plasma virus receiving HAART. Highly active antiretroviral therapy. Lancet 353:119-120.

Neff CP, Ndolo T, Tandon A, Habu Y, and Akkina R (2010) Oral pre-exposure prophylaxis by anti-retrovirals raltegravir and maraviroc protects against HIV-1 vaginal transmission in a humanized mouse model. PLoS One 5: e15257.

Nicol MR, Fedoriw Y, Mathews M, Prince HMA, Patterson KB, Geller E, Mollan K, Mathews S, Kroetz DL, and Kashuba ADM (2014) Expression of six drug transporters in vaginal, cervical, and colorectal tissues: implications for drug disposition in HIV prevention. $J$ Clin Pharmacol 54:574-583.

North TW, Higgins J, Deere JD, Hayes TL, Villalobos A, Adamson L, Shacklett BL, Schinazi RF, and Luciw PA (2010) Viral sanctuaries during highly active antiretroviral therapy in a nonhuman primate model for AIDS. $J$ Virol 84 $2913-2922$

Ohtsuki S, Schaefer O, Kawakami H, Inoue T, Liehner S, Saito A, Ishiguro N, Kishimoto W, Ludwig-Schwellinger E, Ebner T, et al. (2012) Simultaneous absolute protein quantification of transporters, cytochromes P450, and UDPglucuronosyltransferases as a novel approach for the characterization of individual human liver: comparison with mRNA levels and activities. Drug Metab Dispos 40: $83-92$.

Patterson KB, Prince HA, Kraft E, Jenkins AJ, Shaheen NJ, Rooney JF, Cohen MS, and Kashuba ADM (2011) Penetration of tenofovir and emtricitabine in mucosal tissues: implications for prevention of HIV-1 transmission. Sci Transl Med $\mathbf{3}$ 112 re 4

Samji H, Cescon A, Hogg RS, Modur SP, Althoff KN, Buchacz K, Burchell AN, Cohen M, Gebo KA, Gill MJ, et al.; North American AIDS Cohort Collaboration on Research and Design (NA-ACCORD) of IeDEA (2013) Closing the gap: increases in life expectancy among treated HIV-positive individuals in the United States and Canada. PLoS One 8:e81355

Schmittgen TD and Livak KJ (2008) Analyzing real-time PCR data by the comparative C(T) method. Nat Protoc 3:1101-1108.

Shytaj IL, Norelli S, Chirullo B, Della Corte A, Collins M, Yalley-Ogunro J, Greenhouse J, Iraci N, Acosta EP, Barreca ML, et al. (2012) A highly intensified ART regimen induces long-term viral suppression and restriction of the viral reservoir in a simian AIDS model. PLoS Pathog 8:e1002774.

Solas C, Lafeuillade A, Halfon P, Chadapaud S, Hittinger G, and Lacarelle B (2003) Discrepancies between protease inhibitor concentrations and viral load in reservoirs and sanctuary sites in human immunodeficiency virus-infected patients. Antimicrob Agents Chemother 47:238-243.

Speck RR, Yu X-F, Hildreth J, and Flexner C (2002) Differential effects of p-glycoprotein and multidrug resistance protein-1 on productive human immunodeficiency virus infection. J Infect Dis 186:332-340.

Srinivas N (2018) Distribution of Antiretrovirals within the Brain Tissue and Relationship with Neurocognitive Impairment Due to HIV. Doctoral Dissertation, University of North Carolina, Chapel Hill, NC.

Srinivas N, Rosen EP, Gilliland WM Jr, Kovarova M, Remling-Mulder L, De La Cruz G, White N, Adamson L, Schauer AP, Sykes C, et al. (2018) Antiretroviral concentrations and surrogate measures of efficacy in the brain tissue and CSF of preclinical species. Xenobiotica DOI: 10.1080/00498254.2018.1539278 [published ahead of print].

Thompson CG (2017) Tissue Reservoir of HIV: Implications for Eradication. Doctoral Dissertation, University of North Carolina, Chapel Hill, NC.

Thompson CG, Bokhart MT, Sykes C, Adamson L, Fedoriw Y, Luciw PA, Muddiman DC, Kashuba ADM, and Rosen EP (2015) Mass spectrometry imaging reveals heterogeneous efavirenz distribution within putative HIV reservoirs. Antimicrob Agents Chemother 59:2944-2948.

Thompson CG, Fallon JK, Mathews M, Charlins P, Remling-Mulder L, Kovarova M, Adamson L, Srinivas N, Schauer A, Sykes C et al (2017) Multimodal analysis of drug transporter expression in gastrointestinal tissue. AIDS 31 1669-1678. 
Van Loo PLP, Van Zutphen LFM, and Baumans V (2003) Male management: coping with aggression problems in male laboratory mice. Lab Anim 37: $300-313$.

Veselinovic M, Yang K-H, LeCureux J, Sykes C, Remling-Mulder L, Kashuba ADM, and Akkina R (2014) HIV pre-exposure prophylaxis: mucosal tissue drug distribution of RT inhibitor tenofovir and entry inhibitor maraviroc in a humanized mouse model. Virology 464-465:253-263.

Viread. (2001) Package insert. Gilead Sciences Inc., Foster City, CA.

Zhou T, Hu M, Cost M, Poloyac S, and Rohan L (2013) Short communication: expression of transporters and metabolizing enzymes in the female lower genita tract: implications for microbicide research. AIDS Res Hum Retroviruses 29 : 1496-1503.

Address correspondence to: Dr. Angela D.M. Kashuba, Division of Pharmacotherapy and Experimental Therapeutics, Eshelman School of Pharmacy, University of North Carolina at Chapel Hill, 1094 Genetic Medicine Building, CB\#7361, 120 Mason Farm Rd., Chapel Hill, NC 27599-7569. E-mail: akashuba@unc.edu 